\title{
Studying Dopamine in Addiction: The Cart Should Follow the Horse
}

Terry E. Robinson ${ }^{1 *}$, Shaun Y-S. Khoo², Carrie R. Ferrario ${ }^{1,3}$ and Anne-Noël Samaha ${ }^{2 *}$

${ }^{1}$ Department of Psychology (Biopsychology), The University of Michigan, Ann Arbor, MI, USA 48109;

2Department of Pharmacology and Physiology, Faculty of Medicine, Université de Montréal, Montréal, QC, Canada H3C 3J7;

${ }^{3}$ Department of Pharmacology, The University of Michigan, Ann Arbor, MI, USA 48109

*Correspondence: ter@umich.edu (Terry Robinson) or anna.samaha@umontreal.ca (AnneNoël Samaha)

Keywords: Addiction, Animal Models, Cocaine Self-Administration, Dopamine, Glutamate plasticity.

(C) 2021. This manuscript version is made available under the CC-BY-NC-ND 4.0 license https://creativecommons.org/licenses/by-nc-nd/4.0/ in accordance with Elsevier's Article Sharing policy.The final published version is available at: https://doi.org/10.1016/j.tins.2021.06.008

The commentary by Lüscher and Pascoli [1] appropriately expands the scope of possible mechanisms by which exposure to drugs of abuse may produce long-lasting changes in brain and behavior. Their commentary focuses on the role of dopamine (DA) as a modulator of other neurotransmitters such as glutamate and GABA. They highlight the ability of these amino acid transmitters to engage intracellular signaling cascades that produce various forms of synaptic plasticity, thereby altering circuit function in ways that may contribute to long-term changes in behavior related to addiction. For example, many studies have examined how glutamate plasticity produced by drug self-administration under long-access conditions contributes to 'relapse' and 'craving' in animal models [2-4]. We completely agree that forms of 
drug-induced plasticity that may contribute to addiction do not reflect a simple up or down in DA neurotransmission, but complex changes in synaptic transmission and neural circuitry ('sideways' in the Lüscher and Pascoli commentary) that mediate motivation for drug and many other psychological processes, such as cognitive control [5]. In our article [6] we cautioned that we would not address the complexity of this circuitry, as Lüscher and Pascoli [1] rightly do.

However, it is worth noting that none of the studies on drug- or DA-induced plasticity cited by Lüscher and Pascoli [1] involved procedures that are effective in producing the addictionrelevant patterns of drug-seeking and -taking behaviors that are seen, for instance, with the intermittent self-administration procedures discussed in our article. Instead, these studies often examined forms of synaptic plasticity produced by a few intraperitoneal injections of cocaine (or sometimes a single injection), or, occasionally, short-access self-administration procedures, which again, do not produce the same behavioral outcomes as either the long- or intermittent access procedures we discussed (cf. [7]). Nevertheless, work - including studies by Lüscher and colleagues - has shown that all these manipulations (and others) produce a variety of forms of synaptic plasticity that can be related to changes in behavior. Indeed, by using such procedures one may learn about how drugs can change the brain and produce associated changes in drug-mediated behaviors such as renewed drug-seeking. However, a central point of our article was to question whether the forms of drug-induced plasticity identified in such studies tell us much about the drug induced changes in brain that are responsible for the transition from casual patterns of drug use to addiction [7].

In our article we argued that if the goal of preclinical studies of addiction is to identify changes in the brain that pathologically increase motivation for drug, or produce other behavioral features of addiction, one should use procedures that most closely mimic patterns of human drug use and that are effective in producing these features [6]. As stated by Niv, 'If you are interested in understanding the provenance of behavior ... you should study behavior' [8]. Thus, the behavioral models used are just as important as the techniques used to examine alterations in neural function. In our article we reviewed evidence that even the two self-administration procedures (long and intermittent access to drug), that are thought to be the most effective in producing signs of addiction, have very different effects on DA function (the 'ups' and 'downs'). This highlights the complexity of the problem - if two self-administration procedures are both thought to model addiction, but produce opposite effects on DA function, the field clearly needs to grapple with what it seeks a mechanism for [7]. 
Finally, we would like to re-emphasize another key point of our article, which concerns the primacy of the behavior or psychological function for which one seeks a cellular mechanism(s). Although this point has been made many times over the years (e.g., [9]), it is too often overlooked and needs to be reiterated. As put by Krakauer et al. [10], 'Insofar as the goal of a neuroscience research question is to explain some behavior ... the behavioral research must be considered, for the most part, epistemologically prior', and 'study of the neural implementation of behavior is best investigated after such behavioral work'. Or as also put by Niv [8], "For the sake of better understanding the brain, we should therefore reverse the current 'hierarchy' (in which neural measurements are seen as basic and fundamental, and behavior is an optional component that cannot stand on its own) and restore behavioral research to its historical place of primacy and necessity." However, in addiction research [11] and other fields (e.g., [12]) the rush to seek mechanisms often results in a tendency to put the cart before the horse. The fact that there is much technologically sophisticated neuroscience research using overly simplistic behavioral endpoints may be one factor contributing to the difficulty in translating such research to the human condition. We suggest, therefore, that progress is going to require avoiding a rush to reductionism and putting the horse and cart in their proper locations. 


\section{References}

1. Luscher, C. and Pascoli, V. (2021) 'Ups, downs and sideways' of dopamine in drug addiction. Trends Neurosci. 44, 593-594.

2. Dong, Y. et al. (2017) Circuit and Synaptic Plasticity Mechanisms of Drug Relapse. J Neurosci 37 (45), 10867-10876.

3. Wolf, M.E. (2016) Synaptic mechanisms underlying persistent cocaine craving. Nat Rev Neurosci 17 (6), 351-65.

4. Scofield, M.D. et al. (2016) The Nucleus Accumbens: Mechanisms of Addiction across Drug Classes Reflect the Importance of Glutamate Homeostasis. Pharmacol Rev 68 (3), 816-71.

5. Sarter, M. and Phillips, K.B. (2018) The neuroscience of cognitive-motivational styles: Signand goal-trackers as animal models. Behav Neurosci 132 (1), 1-12.

6. Samaha, A.N. et al. (2021) Dopamine 'ups and downs' in addiction revisited. Trends Neurosci. 44, 516-526.

7. Kawa, A.B. et al. (2019) The transition to cocaine addiction: the importance of pharmacokinetics for preclinical models. Psychopharmacology (Berl) 236 (4), 1145-1157.

8. Niv, Y. (2021) The primacy of behavioral research for understanding the brain. Behav Neurosci. doi: 10.1037/bne0000471

9. Robinson, T.E. (Ed.) (1983) Behavioral Approaches to Brain Research, Oxford University Press.

10. Krakauer, J.W. et al. (2017) Neuroscience Needs Behavior: Correcting a Reductionist Bias. Neuron 93 (3), 480-490.

11. Kalant, H. (2010) What neurobiology cannot tell us about addiction. Addiction 105 (5), 780 9.

12. Pare, D. and Quirk, G.J. (2017) When Scientific Paradigms Lead to Tunnel Vision: Lessons from the Study of Fear. NPJ Sci Learn 2: 6. 